\title{
UNA VISIÓN MÁS REALISTA DE LA CONVENCIÓN SOBRE LOS DERECHOS DE LAS PERSONAS CON DISCAPACIDAD
}

\author{
Sergio Ramos Pozón ${ }^{1}$
}

Resumen: En este artículo analizamos de manera crítica la Convención sobre los derechos de las personas con discapacidad. Una visión más realista puede ser llevada a cabo cuando se aplica a personas con enfermedad mental. Dos situaciones clínicas serán revisadas a la luz de la Convención: el consentimiento informado y las decisiones por representación. Bajo esta interpretación se propone que la aplicación de la Convención en salud mental respetará satisfactoriamente los derechos humanos de las personas con discapacidad.

Palabras clave: CDPD, consentimiento informado, decisiones por sustitución, ingreso involuntario, derechos humanos

\section{A realistic view of the Convention on the Rights of Persons with Disabilities}

Abstract: In this article, we critically analyze the Convention on the Rights of Persons with Disabilities. A realistic view should be taken into account when the text is applied in persons with mental illness. Two clinical situations will be revised in light of the Convention: informed consent and substitute decision-making. This interpretation states that the application of the Convention in Mental Health will respect satisfactory human rights of persons with disability.

Key words: CRPD, informed consent, substitute decision-making, involuntary treatment, human rights

\section{Uma visáo mais realista da Convençáo sobre os direitos das pessoas com deficiência}

Resumo: Neste artigo analisamos criticamente a Convenção sobre os direitos das pessoas com deficiência. Uma visão mais realista pode ser feita quando se aplica a pessoas com doença mental. Duas situaçóes clínicas serão revistas à luz da Convenção: o consentimento informado e decisóes pela representação. Sob esta interpretação, propõe-se que a implementação da Convenção em Saúde mental respeitará satisfatoriamente os direitos humanos das pessoas com deficiência.

Palavras-chave: CDPD, consentimento informado, decisôes pela substituição, admissão involuntária, direitos humanos

\footnotetext{
${ }^{1}$ Universidad de Barcelona, Espańa

Correspondencia: ramospozon@hotmail.com
} 


\section{Introducción}

En 2006 las Naciones Unidas llevaron a cabo el proyecto sobre la Convención sobre los derechos de las personas con discapacidad (CDPD)(1), entrando en vigor en 2008. Es el primer tratado internacional con carácter exclusivo en materia de derechos humanos para personas con discapacidad. Propone un conjunto de derechos humanos, civiles, político-sociales, económicos y culturales.

El propósito consiste en "promover, proteger y asegurar el goce pleno y en condiciones de igualdad de todos los derechos humanos y libertades fundamentales por todas las personas con discapacidad, y promover el respeto de su dignidad inherente" (1, art. 1).

Aunque se trata de una legislación que mejora significantemente los derechos de estas personas y obliga a los Estados partes a su cumplimiento, la realidad muestra que el texto no ha sido lo suficientemente incorporado en las legislaciones de dichos Estados. En particular, la Convención observó que hay un malentendido en el alcance de las obligaciones referentes al artículo 12 sobre el igual reconocimiento ante la ley. Con este objetivo, el Comité realizó una Observación General(2) concerniente al artículo 12 (y artículos vinculados), con fuertes comentarios en el ámbito de la salud mental.

En esta Observación se clarifica y regula la toma de decisiones, ya que se insta a llevar a cabo un "cambio de paradigma" que pase del tradicional modelo de sustituciones a uno basado en el soporte para que sea la persona quien $\operatorname{decida}(3,4)$. El problema, sin embargo, es que tal enfoque no suele ser fácilmente aplicado a todas las personas con discapacidad, ya que su aplicabilidad va en función de sus condiciones bio-psico-sociales(5).

Este cambio de paradigma significa una potestad para que sea la persona con discapacidad quien escoja libremente. Ese igual reconocimiento supone el respeto por la dignidad y la autonomía, lo que implica que los Estados partes tienen que examinar sus leyes y políticas que regulan la guarda y la tutela para fomentar un modelo basado en tal soporte a las decisiones y garantizar su voluntad y preferencias(2, para. 26). Además, se anima a im- plantar "salvaguardias" para garantizar ese respeto (2, para. 4).

Ahora bien, algunos puntos del texto y, especialmente la Observación General, deberían ser revisados o fortalecidos para luchar por los derechos de estas personas, especialmente las que padecen de enfermedad mental.

\section{Método}

Este trabajo revisa críticamente la Convención sobre los derechos de las personas con discapacidad. En especial, se enfoca en el ámbito de la salud mental, entendida como discapacidad. Para ello, se analizan dos situaciones clínicas que desafían el texto de la Convención: el consentimiento informado y las decisiones por sustitución. El objetivo es examinar algunos puntos clave que pueden ser cuestionables cuando aplicamos la Convención a personas con enfermedad mental. Nos apoyamos en normas jurídicas, argumentos éticos y datos empíricos.

\section{Desafíos y consideraciones en torno a la Convención}

La CDPD presenta un tratado muy positivo para la implantación de los derechos humanos. Aunque es necesario dar "protagonismo" a estas personas en relación con las decisiones referentes a su vida, hay contextos clínicos significativos que hacen dudar de dicha aseveración, e incluso se pone en duda que se trate del mayor beneficio para la persona. Un excesivo peso en la autonomía en aras de otros principios podría poner en juego la vida de la persona. Por tanto, aunque el ideal es un modelo de relación asistencial en el que el paciente decida(6), en ocasiones algunas ciertas formas de "paternalismo" podrían justificarse. Esto no ha de ser la regla, sino la excepción.

Con esta pretensión, analizaremos el consentimiento informado (CI) y las decisiones por representación. Se van a revisar a la luz de la Convención con el fin de garantizar que se cumplan siguiendo la esencia del texto.

\section{El consentimiento informado}

Ciertamente, el CI se ha instaurado en el ámbito de la salud con el fin de preservar la autonomía y 
la dignidad del paciente. La Convención promueve un modelo de relación asistencial basado en el soporte para que la persona escoja una línea terapéutica u otra, siendo este el fundamento del CI.

Ahora bien, en salud mental, pese a que los profesionales instan a que sean los pacientes quienes decidan libremente, la investigación ha detectado que la tendencia dominante es el paternalismo(7). No obstante, esta presunción no está fundada empíricamente, ya que los estudios muestran que los pacientes con enfermedades mentales, como por ejemplo los que padecen de esquizofrenia $(8,9)$, sí se interesan en participar y además lo hacen activamente. Por tanto, se tendría que respetar la autonomía siempre y cuando no haya indicios claros de incompetencia (aptitudes y habilidades psicológicas suficientes) que pongan en duda su participación.

El problema es que la Convención no parece reconocer que la competencia es una cuestión médica, sino un constructo social:

La capacidad mental no es, como se presenta comúnmente, un fenómeno objetivo, científico y natural, sino que depende de los contextos sociales y políticos, al igual que las disciplinas, profesiones y prácticas que desempeñan un papel predominante en su evaluación (2, para. 14).

Ahora bien, en este punto discrepamos, pues en la literatura referente a la valoración de la competencia de los pacientes se cita frecuentemente los trabajos de Grisso y Appelbaum(10). Estos autores proponen el MacCAT-T para la valoración y evalúan como parámetros la comprensión, el razonamiento, la apreciación y la elección de una opción terapéutica.

En primer lugar, la comprensión hace referencia a la habilidad de la persona para entender la información que el médico le está proporcionando sobre el diagnóstico de la enfermedad y su tratamiento. Para ver el grado de comprensión se puede pedir que la parafrasee. El razonamiento se centra en el análisis de la habilidad para procesar y analizar la información, ponderando las distintas alternativas terapéuticas en relación a las reacciones adversas y el posible impacto en su vida cotidiana. La apreciación consiste en la capacidad del paciente para relacionar toda esa información con su situación. A fin de cuentas, lo que se pretende es saber si conoce su diagnóstico y si es consciente de que el tratamiento propuesto puede beneficiarle. Finalmente, la expresión de una elección se refiere a la habilidad para comunicar su decisión respecto del tratamiento médico propuesto.

Se trata de una herramienta validada con varios tipos de pacientes, también psiquiátricos(11-13). Por tanto, hay suficiente evidencia empírica para establecer que la competencia es una cuestión científica y objetiva.

Ahora bien, esta evaluación también nos muestra que los pacientes con enfermedades mentales pueden reflejar factores que condicionan negativamente la competencia para la toma de decisiones: la intensidad de la psicopatología, el deterioro cognitivo (en particular en los procesos de atención), un rendimiento intelectual bajo, problemas para el almacenamiento de información, etc.

El análisis de los factores cognitivos $(13,14)$ también demuestra que, aunque pueden tener una peor puntuación en la escala del MacCAT-T, sí que pueden tomar decisiones de manera responsable. Sin embargo, mucho más difícil es en aquellos que no tienen conciencia de enfermedad (insight), razón por la cual consideran que no tienen que tomar la medicación porque "no están enfermos" $(15,16)$. Por tanto, se requiere un análisis riguroso cuando la competencia esté comprometida.

Pero, de nuevo, da la sensación de que la CPDP no tiene en cuenta que el CI suele tener excepciones, como son la incompetencia o las urgencias médicas(17). Por ejemplo, carece de sentido intentar recabar el CI cuando el paciente está en una fase delirante. En tales casos, se justifica una aplicación terapéutica sin el consentimiento del paciente. No obstante, siempre se debe respetar la dignidad y la integridad de la persona (principalmente ante intervenciones terapéuticas coercitivas), y siempre con vistas a un plan terapéutico que tenga en cuenta la búsqueda del mayor beneficio y la evitación de daños para el paciente y terceros. 
En ese punto se debería evaluar los ingresos y los tratamientos involuntarios en aras del bien de la persona, aunque esto siempre es muy controvertido, ya que le priva del derecho a la libertad(18). Pero de nuevo la Convención se pronuncia negativamente y promueve que dichas prácticas han de ser abolidas pues son una negación del igual reconocimiento ante la ley, una violación del derecho a la integridad, libertad y tortura, suponiendo pues un caso de abuso(2, para. 7). No obstante, estos tipos de medidas son frecuentemente utilizadas en salud mental bajo algunas circunstancias específicas. Esto supone un desafío para la CDPD(19-21) Pero si adoptamos dichas medidas, los criterios que se apliquen han de ser neutrales hacia la discapacidad, es decir, basados en razones que no discriminen(21).

En efecto, deberíamos partir de que las personas tienen el derecho a escoger o rechazar tratamientos (respeto por la libertad, voluntad y preferencias), siempre y cuando sean competentes. Esto supone el respeto por la igualdad y la no discriminación. Al pedir ese mínimo de competencia requerida a todas las personas, no se discrimina por motivos de discapacidad o enfermedad, ya que será un trato por igual.

El problema es que frecuentemente se justifica un ingreso o tratamiento involuntario con base en el criterio de la peligrosidad, lo cual discrimina, etiqueta y no siempre es cierto; de manera que sería una mejor estrategia basarse en el criterio de la competencia(22), y siempre con vistas a alcanzar el mayor beneficio para la persona Además, suele ser común argumentar que estas medidas se deberían aplicar cuando hay un peligro para la salud de la persona, por la posibilidad de que realice conductas agresivas o por el deterioro (físico y psicológico) que puede desembocar el no seguimiento farmacológico. Esto es algo que han recogido varias normativas legales(23-25)

En cualquier caso, estas medidas han de usarse siempre apelando al texto de la Convención. Efectivamente, el artículo 10 establece la obligación de los Estados partes de garantizar el derecho a la vida (de calidad). Este derecho se tendría que leer conjuntamente con el de la salud (art. 26), que engloba tanto una identificación precoz como una intervención en casos de enfermedad mental severa que irremediablemente desembocará en diversos tipos de discapacidades (mentales o biológicas debido al impacto de la sintomatología psiquiátrica, etc.). Un ingreso o un tratamiento involuntario proporcionarían tratamiento necesario para la persona, sin el cual su salud puede verse dańada. Por tanto, los Estados tienen la obligación de minimizar y prevenir tales acontecimientos. En salud mental, uno de los objetivos es la rehabilitación y la reintegración de las personas en la sociedad. Esto permitiría que el paciente pudiese obtener el máximo de independencia, un bienestar bio-psico-social y una participación en todos los aspectos de su vida (art. 27).

En conclusión, dichas prácticas tendrían que ser solo una excepción y no una regla. El CI tendría que ser la base de la relación asistencial. Sea como fuere, resulta llamativo que la Convención no tenga en cuentas estas consideraciones que, aunque meramente clínicas, tienen un fuerte componente ético, ya que en algunas situaciones se podría dejar decidir a personas que no están capacitadas para hacerlo y que podría poner en peligro su vida y la de otros.

Pero, además, esta excesiva preocupación porque las personas decidan libremente no permite la posibilidad de que no quieran participar en la toma de decisiones. En efecto, parece comprensible que, incluso siendo competente para decidir, no quiera participar y desee dejar en manos de la familia y/o del equipo médico cualquier decisión sobre su salud $(26,27)$. Respetar la autonomía también significa el derecho a no ser informado y querer que sean otros los que decidan.

Así, las decisiones subrogadas presentan un desafío para la CDPD, pues proponen una relación asistencial basada en el soporte, más que en las sustituciones.

\section{Del "soporte a las decisiones" a las "decisio- nes por sustitución", y viceversa}

Ya hemos comentado que la CDPD promueve un cambio de paradigma que va de las decisiones por sustitución al soporte a las decisiones.

No obstante, no es fácil definir satisfactoriamente qué significa "soporte a las decisiones". En este 
intento, algunos autores sostienen diversas definiciones(28). En primer lugar, puede ser concebido como un procedimiento en el cual una persona explica cuestiones a otra e interpreta las señales y preferencias de la persona a quien va dirigido el proceso. En segundo lugar, se trata de una alternativa al modelo de sustituciones. En particular, se considera un cambio de paradigma legal que reconoce, además, que la persona tiene déficits para la toma de decisiones. En tercer lugar, es considerado como un diferente tipo de decisiones por sustitución, que introduce la voluntad y preferencias de la persona, ya que esta no puede tomar decisiones por sí misma y se requiere buscar su mayor beneficio.

Ciertamente, la Convención (2, para. 26) insta a que se sustituyan los regímenes basados en la sustitución y se oriente la toma de decisiones que respeten la autonomía, la voluntad y las preferencias de la persona. Para ello, el texto (artículo 12) propone la adopción de "salvaguardias" para preservar la autonomía. Sin embargo, de nuevo, no se define qué significan "salvaguardias". Con el objetivo de precisarlo, la literatura ha adoptado varios enfoques(29-31) En el ámbito de la salud mental, varios son los modelos que sirven para salvaguardar la autonomía: la toma de decisiones compartidas(32-34), el documento de voluntades anticipadas (DVA) $(35,36)$ y la planificación anticipada de las decisiones (PAD)(6).

Tanto el DVA como la PAD quedan recogidos en la Observación (2, para. 17), al establecer que a las personas con discapacidad se les debe dar la posibilidad de planificar su futura decisión en igualdad de condiciones que a las demás personas.

Ahora bien, en ocasiones las personas no están en disposición como para escoger libre y autónomamente, incluso asumiendo soportes. En salud mental, muchas veces hay que tomar decisiones por sustitución porque están en una fase delirante, porque las funciones cognitivas están fuertemente comprometidas, etc. Pero al acudir al texto de la Convención vemos que no hay una definición clara sobre qué significa "decisiones por sustitución”. En la Observación General(2), se estipula que pueden adoptar muchas formas como la tutela plena o parcial, y la interdicción judicial. Todas comparten unas características: i) se despoja a la persona de la capacidad jurídica, aunque sea con respecto a una única decisión; ii) puede nombrar al sustituto que tomará las decisiones alguien que no sea la persona concernida y ese nombramiento puede hacerse en contra de su voluntad; y iii) toda decisión adoptada por el sustituto en la adopción de decisiones se basa en lo que se considera el "interés superior" objetivo de la persona concerni$d a$, en lugar de basarse en su propia voluntad y sus preferencias (2, párrafo 27 ).

El objetivo es la obtención del mayor bienestar para la persona, de manera que los criterios han de ser los de la beneficencia y la no-maleficencia más que la autonomía. Este deber puede ser interpretado desde el artículo 25 de la CDPD en el que:

los Estados Partes reconocen que las personas con discapacidad tienen derecho a gozar del más alto nivel posible de salud sin discriminación por motivos de discapacidad. Los Estados Partes adoptarán las medidas pertinentes para asegurar el acceso de las personas con discapacidad a servicios de salud que tengan en cuenta las cuestiones de género, incluida la rehabilitación relacionada con la salud.

Y ese bienestar frecuentemente está condicionado por el estigma y la discriminación asociados a la enfermedad mental. Muchas veces a este tipo de pacientes (quizás más a los que sufren psicosis) se les atribuye características como la imprevisibilidad, la peligrosidad, la incompetencia, la violencia, etc. $(37,38)$. Las falsas creencias sobre la enfermedad mental dan lugar al estigma, el cual se traduce en componentes cognitivos, afectivos y conductuales, tanto para quien es etiquetado como para quien etiqueta, exteriorizados en forma de estereotipos, prejuicios y discriminación $(39,40)$ El problema es que esto repercute negativamente en el hecho de que los pacientes acudan a los servicios de salud(41). Los expertos han detectado varias estrategias para reducir tal discriminación: psicoeducación, contacto e interacción con estos pacientes, empoderar a la persona para que participe en la toma de decisiones, etc. $(42,43)$.

Por tanto, ha de ser una obligación moral intentar reducir esa discriminación para que puedan tener un mayor nivel de salud. Además, el artículo 26 promueve que los Estados partes han de tomar 
medidas apropiadas y efectivas, incluso mediante el apoyo, para que las personas con discapacidad puedan disfrutar de la mayor independencia, capacidad física, mental, social y vocacional, y la inclusión y participación plena en todos los aspectos de la vida cotidiana. Y en el área de la salud esto adquiere mayor protagonismo. Así pues, cuando los pacientes tienen competencia para la toma de decisiones, el consentimiento informado debe ser recabado por los profesionales y el paciente debe tener el derecho de escoger o rechazar líneas terapéuticas. Sin embargo, si carece de competencia, debería tomarse una decisión por representación para proteger a la persona vulnerable.

Pero incluso en ese tipo de decisiones hemos de tomar como criterio básico sus preferencias y deseos. El objetivo, por tanto, reside en el respeto y promoción de la autonomía y la dignidad. No obstante, eso no siempre queda bien especificado o clarificado, de modo que, bajo ciertas circunstancias, se debe tomar decisiones prudentes que intenten respetarle lo máximo posible. En algunos casos, la autonomía ha de ser la guía, en otras la beneficencia y no-maleficencia deberán ser decisivas.

Por esa razón, es necesario ser precavido y articular unas normas de actuación y unas normas de intervención para alcanzar el máximo respeto por el paciente vulnerable.

\subsection{Normas de actuación}

En las decisiones por sustitución hay factores que determinan el modo de actuación. Es posible que tengamos constancia explícita de los deseos de la persona, que conozcamos únicamente algunos valores y preferencias o que no sepamos nada sobre ella. En relación a ello, Buchanan A. y Brock D.(44:98-134) señalan tres líneas de actuación: criterio subjetivo, criterio del juicio sustitutivo y criterio del mejor interés.

Criterio subjetivo: tiene como finalidad aplicar las instrucciones previas que la persona ha manifestado. El paciente debe comunicar a los profesionales sus deseos y preferencias. Se puede hacer de manera oral o escrita. Aquí, pues, se engloba el DVA, la PAD o la toma de decisiones compartidas.
Criterio del juicio sustitutivo: es aquel que una persona competente adopta en nombre de otra que es incompetente. Dicha decisión ha de tener como referencia los deseos y preferencias de la persona incapacitada y no los de quien ha de sustituirle. Se trata de decidir teniendo en cuenta lo que ella decidiría.

Pablo Simón e Inés Barrio(45:100) exponen 3 formulaciones de dicho principio:

- El sustituto ha de decidir lo que una persona razonable competente escogería si se hallara en las mismas condiciones, teniendo en cuenta que entonces sería incapaz.

- El sustituto ha de escoger la misma opción que tomaría el mismo paciente si fuera capaz. Para ello, es preciso tener en cuenta la escala de valores del incapacitado: aspectos psicológicos (miedos, tristezas...) y clínicos (diagnóstico, pronóstico, etc.).

- Se debe realizar el mismo silogismo que utilizaría el propio paciente, y para ello ha de establecer unos valores (premisas) desde los cuales extraer las conclusiones.

Criterio del mejor interés: excluye a la persona de la decisión, buscando solo aquello que más le beneficia. Se aplica cuando no sabemos cómo quiere ser tratada (nunca ha sido competente, no ha proporcionado argumentos serios o si carece de familiares que puedan aportar información).

\subsection{Normas de intervención}

Ahora bien, parece razonable pensar que con estas normas no se resuelve satisfactoriamente el modo las decisiones por representación, pues en ocasiones los representantes no pueden ejercer el cargo adecuadamente, no hay una voluntad expresa del paciente o porque las futuras decisiones pueden ser cuestionables. Por eso, es conveniente adoptar también unas normas de intervención para ayudar en el proceso(44:142-147).

\section{Normas que identifican familias no cualificadas}

Las estructuramos en relación a dos características: a) las que se refieren a la familia y b) las que hacen referencia a la relación con la persona incapacitada. 
Respecto del primer grupo, no resultan buenos sustitutos los que sufren algún tipo de enfermedad psiquiátrica, abuso de sustancias, etc., pues pueden tener dificultades incluso para decidir por ellas mismas o no tomarse en serio la tarea de sustitución. Tampoco aquellos familiares de tercera edad que tengan un bajo nivel cognitivo, no sepan apreciar la valoración riesgo-beneficio de los tratamientos, etc. A su vez, han de descartarse aquellos que hayan dañado o abusado de la persona.

En el segundo grupo hay que descartar a los familiares que no conocen suficientemente ni a la persona ni al manejo de los síntomas psiquiátricos, el tratamiento o las recidivas, pues puede influenciar a que se tomen decisiones no favorables a la persona. Tampoco son buenos sustitutos los que no saben qué funciones han de cumplir o que tienen poca relación con la persona, pues difícilmente sabrán qué valores o preferencias tomar. También rechazar a quienes ven la situación como una carga familiar (46), porque puede incidir a que se tomen decisiones que benefician solo a la familia. $Y$ es que, por ejemplo, un paciente con esquizofrenia puede suponer una carga familiar que repercute en la vida de sus cuidadores: tareas de la casa, relaciones de pareja, familiares y sociales, etc.

Normas que identifican casos especiales que requieren especial vigilancia durante el proceso de toma de decisiones

Hay casos especiales que requieren una mayor supervisión y que se refieren a i) pacientes que son especialmente vulnerables, dada su situación clínica y sus características personales (cognitivas, afectivos, sociales); y ii) decisiones críticas o que pueden tener consecuencias importantes para la persona.

En primer lugar, los pacientes con enfermedades mentales que están en una fase aguda son especialmente vulnerables porque pueden ser incompetentes para decidir. También aquellos que han cronificado los síntomas y que la enfermedad repercute considerablemente en su vida, pudiendo presentar síntomas deficitarios para la vida cotidiana, necesitando apoyo para el cuidado personal, el funcionamiento ocupacional, etc. Es frecuente que estos pacientes tengan déficits cognitivos (atención, memoria y funciones ejecutivas) que dificulten la toma de decisiones. En tales casos hay que prestar atención a qué y por qué motivos un sustituto puede decantarse por una línea terapéutica, ya que la persona puede tener poca participación en el proceso.

En segundo lugar, hemos de analizar las decisiones que impliquen un acortamiento considerable de la vida y que podrían ser evitadas. En pacientes con esquizofrenia el suicidio es una causa importante de mortalidad. Entre el $9 \%$ y el 13\% de ellos se suicidan, lo cual está asociado con depresión y consumo de drogas. Suele darse con más frecuencia cuando la persona está triste, aislada, irritable, etc.(47). Es frecuente que los pacientes psiquiátricos que tienen intención de suicidarse comuniquen la decisión a la familia, directa o indirectamente. En casos en los que aparezca algún indicio, la familia debiera solicitar un ingreso involuntario, asumiendo así todas las decisiones. En el momento de recibir el alta, los familiares han de estar alerta para detectar los signos de aviso e iniciar medidas de control si se requiere(48:132). Más que cuestionar la potestad para decidir en nombre de la persona, lo que se pretende es una actitud anticipatoria que evite esa conducta.

Normas que identifican decisiones tomadas por el sustituto que son cuestionables

Es posible que cuando el sustituto tome decisiones, aquello que está solicitando no sea lo mejor para el incapacitado. Puede darse el caso de que no sea admisible desde un punto de vista médico; que, al aplicar al criterio del mejor interés, un sustituto tome una decisión que todas las personas que le rodean no aceptarían; o que, al emplear un juicio sustitutivo, la decisión tomada no se deduzca de las preferencias de la persona incapacitada o claramente le causaría daño.

Pese a que sean los sustitutos quienes tomen las decisiones subrogadas, esto no les da derecho a solicitar o rechazar cualquier tratamiento. Las líneas terapéuticas han de ser acorde con las normas de buena praxis. Tratamientos más "agresivos", como el tratamiento electroconvulsivo, pueden ser útiles en algunos casos clínicos; sin embargo, tiene algunas complicaciones que deberíamos valorar: afectación de la memoria, confusión e hiper- 
tensión. Ahora bien, también se ha demostrado que tiene efectos positivos en la recuperación de la enfermedad y/o puede evitar conductas suicidas(49,50). Por tanto, una elección de este tipo requiere un análisis detallado sobre los beneficios y los inconvenientes(51), por lo que no se puede dejar libremente a los representantes escoger sin un buen acompańamiento de los profesionales.

\section{Conclusión}

En este artículo se revisa críticamente el texto de la Convención sobre los derechos de las personas con discapacidad (CDPD) porque adolece de ciertas deficiencias. Aunque el ideal es que sean las personas (con y sin discapacidad) las que decidan libremente sobre decisiones terapéuticas (si quieren), en ocasiones se requiere un cierto paternalismo, porque bajo algunas circunstancias los pacientes no son capaces de tomar decisiones por sí solos. El CI adquiere un papel relevante, pero no hay que olvidar que tiene excepciones que invalidan su aplicabilidad. En algunos contextos bastará con un soporte para ayudar a estas personas, en otros se tomarán decisiones por representación o incluso pedir algún tipo de ingreso o tratamiento involuntario. En cualquier caso, dichas actuaciones han de estar siempre fundamentadas en los derechos recogidos en la CDPD para respetar la dignidad y no discriminar a los pacientes.

Es preciso revisar las prácticas y leyes de los Estados partes cuando hacen referencia a pacientes con enfermedades mentales, pues hay una ligera tendencia al paternalismo. Ahora bien, aunque en algunas situaciones puede estar justificado, siempre tendríamos que revisar su aplicabilidad en torno a la Convención para asegurar que se respetan los derechos humanos. Así, no se debería presuponer que los pacientes con enfermedades mentales no sean competentes para decidir, y en tal caso se tendría que demostrar mediante la valoración de la competencia. En algunos casos tendríamos que acompañarles para decidir, en otros simplemente decidir responsablemente por ellos. Y no tendríamos que intentar abolir todas las prácticas de ingresos o tratamientos involuntarios, pues en algunos contextos sería incluso indicado. Ahora bien, se requiere un mayor análisis de dichas prácticas e intentar respetar al máximo el texto de la CDPD.

\section{Referencias}

1. United Nations Committee on the Rights of Persons with Disabilities. Convention on the Rights of Persons with Disabilities. 2006. Disponible en http://www.un.org/disabilities/documents/convention/convoptprot-e.pdf Consultado el 1 de septiembre de 2015.

2. United Nations. Committee on the Rights of Persons with Disabilities. General comment No 1 (2014) Article 12: Equal Recognition before the Law. Disponible en: http://www.ohchr.org/EN/HRBodies/CRPD/Pages/GC.aspx. Consultado el 1 de septiembre de 2015 .

3. Lawson A. The United Nations Convention on the Rights of Persons with Disabilities: New Era or False Dawn? Journal of International Law and Commerce 2007; 34: 563-620.

4. Dhanda A. Legal Capacity in the Disability Rights Convention: Stranglehold of the Past or Lodestar for the Future? Syracuse Journal of International Law and Commerce 2007;34: 429-462.

5. Devi N, Bickenbach J, Stucki G. Moving Towards Substituted or Supported Decision-Making? Article 12 of the Convention on the Rights of Persons with Disabilities. European Journal of Disability Research 2011; 5: 249-264.

6. Ramos S, Robles B. La relación médico-paciente en salud mental: el documento de voluntades anticipadas y la planificación anticipada de las decisiones. Rehabilitación Psicosocial 2015; 12(1): 18-24.

7. Pelto V, Engström K, Engström I. Paternalism, Autonomy and Reciprocity: Ethical Perspectives in Encounters with Patients in Psychiatric in-Patient Care. BMC Medical Ethics 2013; 14: 49.

8. Bernard A, Fischer M, Robert P, et al. Participants with Schizophrenia Retain the Information Necessary for Informed Consent During Clinical Trials. J Clin Psychiatry 2013; 74(6): 622-627.

9. Hamann J, Cohen R, Leucht S, et al. Do Patients with Schizophrenia Wish to be Involved in Decisions About their Medical Treatment? Am J Psychiatry 2005; 162(12): 2382-2384.

10. Grisso T, Appelbaum P. Assessing Competence to Consent to Treatment: A Guide for Physicians and Other Health Professionals. New York: Oxford University Press; 1998.

11. Jeste D, Deep C, Palmer B. Magnitude of Impairment in Decisional Capacity in People with Schizophrenia Compared to Normal Subjects: An Overview. Schizophrenia Bulletin 2006; 32(1): 121-128. 
12. Wong G, Cheuny E, Chen E. Decision-Making Capacity of Inpatients with Schizophrenia in Hong Kong. J Nerv Ment Dis 2005; 193: 316-322.

13. Palmer W, Dunn L, Appelbaum P, et al. Correlates of Treatment-Related Decision-Making Capacity among MiddleAge and Older Patients with Schizophrenia. Arch. Gen. Psychiatry 2004; 61: 230-236.

14. Wong J, Clare C, Holland A, et al. The Capacity of People with a 'Mental Disability' to Make a Health Care Decision. Psychol Med 2000; 30(2): 295-306.

15. Capdevielle D, Raffard S, Bayard S, et al. Competence to Consent and Insight in Schizophrenia: Is there an Association? A Pilot Study. Schizophrenia Research 2009; 108(1-3): 272-279.

16. Bitter I, Fehér L, Tényi T, et al. Treatment Adherence and Insight in Schizophrenia. Psychiatria Hungarica 2015; 30(1): 18-26.

17. Ramos S. Elementos necesarios al consentimiento informado en pacientes con esquizofrenia. Revista de Bioética 2015; 23(1): 20-30.

18. Kelly B. An End to Psychiatric Detention? Implications of the United Nations Convention on the Rights of Persons with Disabilities. The British Journal of Psychiatry 2014; 204: 174-175.

19. Dawson J. A Realistic Approach to Assessing Mental Health Law's Compliance with the UNCRPD. International Journal of Law and Psychiatry 2015; 40: 70-79.

20. Colin M, Kolappa K, Caldas J, et al. Reversing Hard Won Victories in the Name of Human Rights: a Critique of the General Comment on Article 12 of the UN Convention on the Rights of Persons with Disabilities. Lancet Psychiatry 2015 Doi: http://dx.doi.org/10.1016/S2215-0366(15)00218-7.

21. Szmukler G, Daw R, Callard F. Mental Health and the UN Convention on the Rights of Persons with Disabilities. International Journal of Law and Psychiatry 2014; 37: 245-252.

22. Large M, Ryan C, Nielssen O, et al. The Danger of Dangerousness: Why we must Remove the Dangerousness Criterion from our Mental Health Acts. Journal of Medical Ethics 2008; 34: 877-881.

23. Recommendation No. REC (2004) 10 of the Committee of Ministers to member States concerning the protection of the human rights and dignity of persons with mental disorder. Disponible en: https://wcd.coe.int/ViewDoc.jsp?id=775685. Consultado el 1 de setiembre de 2015.

24. Convention for the Protection of Human Rights and Dignity of the Human Being with Regard to the Application of Biology and Medicine. 1997. Disponible en: http://www.unav.es/cdb/coeconvencion.html. Consultado el 1 de setiembre de 2015.

25. World Psychiatric Association. Declaration of Hawaii. Disponible en http://www.ncbi.nlm.nih.gov/pmc/articles/ PMC1154636/pdf/jmedeth00167-0017.pdf. Consultado el 1 de setiembre de 2015.

26. Mckinstry B. Do Patients Wish to be Involved in Decision Making in the Consultation? A Cross Sectional Survey with Video Vignettes. BMJ 2000; 321: 867-871.

27. Levinson W, Kao A, Kudy A, et al. Not all Patients Want to Participate in Decision Making. A National Study of Public Preferences. J Gen Intern Med 2005; 20: 531-535.

28. Browning M, Bigby Ch, Douglas J. Supported Decision Making: Understanding How its Conceptual Link to Legal Capacity is Influencing the Development of Practice. Research and Practice in Intellectual and Developmental Disabilities 2014; 1(1): 34-45.

29. Then S. Evolution and Innovation in Guardianship Laws: Assisted Decision-Making. Sydney Law Review 2013; 35: 133-166.

30. Pathare S, Shields L. Supported Decision-Making for Persons with Mental Illness: A Review. Public Health Review 2012; 34(2): 1-40.

31. Browning M. Report by Michelle Browning 2010 Churchill Fellow to Investigate New Models of Guardianship and the Emerging Practice of Supported Decision Making. Canberra: Winston Churchill Memorial Trust; 2010.

32. Hamann J, Leucht S, Kissling W. Shared Decision-making in Psychiatry. Acta Psychiatr Scand 2003; 107: $403-409$.

33. Hamann J, Mendel R, Fink B, et al. Patients' and Psychiatrists' Perceptions of Clinical Decisions during Schizophrenia Treatment. J Nerv Ment Dis 2008; 196: 329-332.

34. Ramos S. La toma de decisiones compartidas en pacientes con esquizofrenia: cuestiones médicas y éticas. Dilemata. Revista Internacional de Eticas Aplicadas 2012; 10: 263-277.

35. Ramos S, Román B. Las voluntades anticipadas en pacientes con esquizofrenia: un instrumento para potenciar la autonomía. Revista de la Asociación Española de Neuropsiquiatría 2014; 34(121): 21-35.

36. Ramos S. Las voluntades anticipadas en salud mental: hechos y valores. Revista de Psiquiatría y Salud mental 2015. Doi: http://dx.doi.org/10.1016/j.rpsm.2015.04.001.

37. Angermeyer MC, Dietrich S. Public Beliefs about and Attitudes towards People with Mental llness: a Review of Popula- 
Una visión más realista de la Convención sobre los derechos de las personas con discapacidad - Sergio Ramos Pozón tion Studies. Acta Psychiatr Scand 2006; 113: 163-179.

38. Angermeyer MC, Matschinger H. The Stigma of Mental Illness: Effects of Labelling on Public Attitudes towards People with Mental Disorder. Acta Psychiatr Scand 2003; 108: 304-309.

39. Corrigan P, Watson A. Understanding the Impact of Stigma on People with Mental Illness. World Psychiatry 2001; 1 : 16-20.

40. Corrigan P, Watson A. Understanding the Impact of Stigma on People with Mental Illness. World Psychiatry 2001; 1: 16-20.

41. Corrigan P, Druss B, Perlick D. The Impact of Mental Illness Stigma on Seeking and Participating in Mental Health Care. Psychological Science Interest 2014; 15(2): 37-70.

42. Mittal D, Sullivan G, Chekuri L, et al. Empirical Studies of Self-Stigma Reduction Strategies: A Critical Review of the Literature. Psychiatric Services 2012; 63(10): 974-981.

43. Corrigan P, River L, Lundin R, et al. Three Strategies for Changing Attributions about Severe Mental Illness. Schizophrenia Bulletin 2001; 27(2): 187-195.

44. Buchanan A, Brock D. Deciding for Others. The Ethics of Surrogate Decision Making. New York: Cambridge University Press; 1989.

45. Simón P, Barrio I. ¿Quién decidirá por mí? Ética de las decisiones clinicas en pacientes incapaces. Madrid: Triacastela; 2004.

46. Martínez A, Nadal S, Beperet M, et al. Sobrecarga de los cuidadores familiares con esquizofrenia: factores determinantes. Anales Sis San Navarra 2000; 23(1): 101-110.

47. Rascón M, Gutiérrez M, Valencia M, et al. Percepción de los familiares del intento de ideación suicidas de pacientes con esquizofrenia. Salud Mental 2004; 27(5): 44-52.

48. Grupo de trabajo de la Guía de Práctica Clínica sobre la Esquizofrenia y el Trastorno Psicótico Incipiente. Fòrum de Salut Mental, coordinación. Guía de Práctica Clínica sobre la Esquizofrenia y el Trastorno Psicótico Incipiente. Madrid: Plan de Calidad para el Sistema Nacional de Salud del Ministerio de Sanidad y Consumo. Agència d'Avaluació de Tecnologia i Recerca Mèdiques; 2009.

49. Kujala I, Rosenvinge B, Bekkelund S. Clinical Outcome and Adverse Effects of Electroconvulsive Therapy in Elderly Psychiatric Patients. J Geriatr Psychiatry Neuro 2002; 15(2): 73-76.

50. MacQueen G, Parkin C, Marriott M, et al. The Long-term Impact of Treatment with Electroconvulsive Therapy on Discrete Memory Systems in Patients with Bipolar Disorder. J Psychiatry Neurosci 2007; 32(4): 241-249.

51. Sackeim H, Prudic J, Fuller R, et al. The Cognitive Effects of Electroconvulsive Therapy in Community Settings. Neuropsychopharmacology 2007; 32: 244-254.

Recibido: 22 de junio de 2015

Aceptado: 17 de octubre de 2015 